\title{
Evaluación cualitativa de extractos macerados con diferentes solventes para la extracción de glicoalcaloides y flavonoides de Dioscórea bulbifera en condiciones estándar obtenidos en los llanos orientales de Colombia $^{1}$
}

\section{Qualitative evaluation of extracts macerated with different solvents for extraction of glycoalkaloids and flavonoids from Dioscórea bulbifera in standard conditions obtained in the eastern plains of Colombia}

\author{
J. E. Armenta, D. A. Bejarano, L. G. López y M. A. Cruz
}

Recibido: abril 23 de 2020 - Aceptado: diciembre 26 de 2020

\begin{abstract}
Resumen- Se evaluaron cualitativamente diferentes solventes para la extracción de núcleos esteroides y flavonoides en bulbos de Dioscorea bulbifera Salv. Se analizó el bulbo crudo y macerados en agua, aceite y alcohol 40\%. La triangulación de las observaciones en las RxSh y RxL-B con un patrón químico definido, un blanco y la prueba experimental evaluó la presencia del núcleo químico. Se estableció por matriz de relaciones que el alcohol tuvo mayor afinidad a compuestos flavonoides y alcaloides detectándose que el $\mathbf{1 0 0 \%}$ de ensayos realizados fue positivo para cada núcleo. Se detectó en un $83 \%$ compuestos
\end{abstract}

${ }^{1}$ Producto derivado del proyecto de investigación "Evaluación del contenido de glicoalcaloides y flavonoides totales con diferentes tratamientos de "papa de aire" (Dioscórea bulbifera Salv.) cosechados en los llanos orientales de Colombia". Presentado por el Grupo de Investigación en Ciencia, Tecnología e Innovación Agroindustrial CITIA y el Semillero de Investigación en Agroindustria y Desarrollo, de la Escuela de Ciencias Agrícolas, Facultad de Ciencias Agropecuarias y Recursos Naturales FCARN, de la Universidad de los Llanos-UNILLANOS, Villavicencio, Colombia.

J. E. Armenta, Universidad de los Llanos, Villavicencio, Colombia, email: jhon.armenta@unillanos.edu.co.

D.A. Bejarano, Universidad de los Llanos, Villavicencio Colombia, email: diego.bejarano.navas@unillanos.edu.co.

L. G. López, Universidad de los Llanos, Villavicencio Colombia, email: llopezm@unillanos.edu.co.

M. A. Cruz, Universidad de los Llanos, Villavicencio Colombia, email: maria.cruz@unillanos.edu.co.

Como citar este artículo: Armenta, J. E., Bejarano, D. A., López, L. G. y Cruz, M. A. Evaluación cualitativa de extractos macerados con diferentes solventes para la extracción de glicoalcaloides y flavonoides de Dioscórea bulbifera en condiciones estándar obtenidos en los llanos orientales de Colombia, Entre Ciencia e Ingeniería, vol. 14, no. 28, pp. 59-65, juliodiciembre, 2020. DOI: https://doi.org/ 10.31908/19098367.2017. alcaloides en muestras de bulbo crudo y superior al $70 \%$ para flavonoides. El aceite mineral presentó características conservantes del bulbo $y$ resultados negativos para ambos núcleos. Se concluye que el alcoholato con bulbos de $D$. bulbifera extrae compuestos de núcleos flavonoides y esteroides para emplearse como ingrediente bioactivo para aplicación en diferentes áreas industriales.

Palabras clave- Extracción, Alcaloides, Flavonoides, Macerados, Detección química, Liebermann-Burchard, Shinoda.

Abstract - Different solvents were qualitatively evaluated for the extraction of steroid nuclei and flavonoids in Dioscorea bulbifera Salv. bulbs. The raw bulb was analyzed and macerated in water, oil, and $40 \%$ alcohol. The triangulation of the observations in the RxSh and RxL-B with a defined chemical pattern, a target, and the experimental test evaluated the presence of the chemical nucleus. The relationship matrix analyzed that the alcohol had a higher affinity to flavonoid and alkaloid compounds, detecting that $100 \%$ of the tests carried out were positive for each nucleus. Alkaloid compounds were detected in $83 \%$ in raw bulb samples and more than $70 \%$ for flavonoids. Mineral oil contains preservative characteristics of the bulb and negative results for both nuclei. It is concluded that the bulbous alcoholate of $D$. bulbifera extracts compounds from flavonoid nuclei and steroids to be used as a bioactive ingredient for application in different industrial areas.

Keywords - Extraction, Alkaloids, Flavonoids, Macerated, Chemical Detection, Liebermann-Burchard, Shinoda.

\section{NOMENCLATURA}

D. bulbifera $=$ Dioscórea bulbifera Salv.

$\mathbf{R x S h}=$ Reacción Shinoda

RxL-B $=$ Test de Liebermann y Burchard

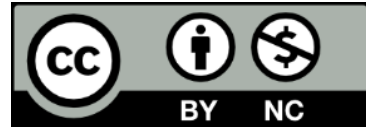

Attribution-NonCommercial 4.0 Intenational (CC By-NC 4.0) 


\section{INTRODUCCIÓN}

T a papa de aire como se le conoce comúnmente a la $D$. Lulbifera es un bulbo aéreo de la familia Dioscórea similar al ñame común (Dioscórea esculenta). Estos vegetales, característicos de clima caliente húmedo y tropical se encuentra distribuido extensamente en Asia y África. La morfología de las variedades encontradas en América en estado silvestre podría dar indicio de su origen. De acuerdo con los estudios en Estados Unidos, sugieren ser una variedad proveniente de África por su forma angular o subangular[1], ([2] página 538).

Existen variedades silvestres de algunos ñames en especial del género Dioscorea que tienen a menudo sustancias venenosas, refiriéndose a los glicoalcaloides ([2] página 1). Los alimentos poseen sustancias nocivas que pueden generar infecciones e intoxicaciones, pero estas consecuencias están en función de la dosis ([3]pagina 197). El compuesto esteroidal se torna tóxico cuando las concentraciones son superiores a 5 $\mathrm{mg} / \mathrm{kg}$ de masa corporal, dosis más pequeñas actúan como inhibidores contra células malignas[4] y como bloqueadores para controlar algunos microorganismos por sus propiedades venenosas ([2]página 538).

Los glicoalcaloides son considerados como metabolitos indicadores de estrés vegetal biótico ([5] página 195, 196) de suma importancia en el control de efectos patológicos causados por plagas. La solanina es un glicoalcaloide común de la familia de las Solanácea como la papa común (Solanun Tuberosum), de igual manera, especies de la familia Dioscoreaceae también contienen compuestos de esta naturaleza como la saponina[3]; naturalmente se encuentra en diversas partes de la planta: hojas, tallos, tubérculos y bulbos, especialmente son mecanismo de defensa instintivo frente a hongos y pesticidas ([4] página 196).

Los flavonoides en bulbos del género Dioscórea son importantes para la supervivencia de la planta, puesto que están encargados del transporte de auxinas, inhibición, protección ultravioleta (UV) y la alelopatía frente a factores externos como la radiación[5]. Los bulbos de D. bulbifera al tener un desarrollo aéreo, son proclives a sintetizar y acumular gran cantidad de metabolitos secundarios, como los flavonoides que se forman en respuesta al estímulo y estrés fisiológico [6]. Una ingesta de alimentos rica en flavonoides aumenta la capacidad antioxidante humana y favorece la atenuación de enfermedades inflamatorias y problemas como daño tisular producida por infección de patógenos [7].

Las variedades silvestres como $D$. bulbifera estudiadas, son más propensas a desarrollar una mayor producción de compuestos polifenólicos como medio defensivo ante agentes externos[8]. La quercitina es uno de los flavonoles con mayor presencia en frutas y vegetales [9]. Sin embargo, en variedades como la $D$. bulbifera los flavonoides representan el $39,6 \%$ y se han encontrado más de 20 tipos de flavonoides, algunos son, 3,5-dimethoxykaempferol, 3,5,30-trimethoxyquercetin, myricetin-3-O-b-D-galactopyranoside, entre otro [10]. La creciente evidencia científica ha demostrado que los compuestos polifenólicos, como los flavonoides, que se encuentran en el reino vegetal, tienen propiedades antiinflamatorias y antioxidantes [7]. Debido a esto, el interés por detectar la presencia de compuestos de esta naturaleza en los bulbos de $D$. bulbifera impulsaría el consumo potencial aplicado en áreas alimentarias y de la salud [11].

Los solventes seleccionados comparten unas serie de características, dentro de las cuales es deseable que sean de fácil adquisición, abundancia en un ambiente rural y citadino, de bajo costo y por efecto de la polaridad se designó el alcohol etílico disuelto al $40 \%$ adaptado de estudios adelantados en la referencia [12], que modelan un experimental para la extracción de compuestos glicoalcaloides y flavonoides, usando como solvente soluciones ácidas de etanol. El aceite mineral al ser inodoro e incoloro es factible para evidenciar la extracción, existen muchos usos comerciales de estos aceites minerales como aditivos alimentarios. El agua es el solvente universal además que, estudios etnobotánicos en Panamá afirman que culturas rurales preparan alimentos con bulbos del género Dioscórea remojados en agua por 12 horas[13]. En los últimos años ha aumentado el número de estudios que afirman tener interés por el desarrollo de bioprocesos para la producción o extracción de compuestos bioactivos de fuentes naturales[10], [14], dadas las posibles aplicaciones de estos compuestos en la industria alimentaria.

Para la extracción, es necesario hacer lisis de los tejidos vegetales como la pared celular, firme y compleja de penetrar por el solvente, una manera fácil de lograrlo es mediante la maceración. Esta técnica permite que el solvente (fase líquida) penetre en los tejidos de la muestra vegetal (fase sólida) provocando que se ablande $y$ se liberen porciones solubles[11], [12].

La finalidad de la investigación es evaluar cuál solvente permite por medio de maceración, separar compuestos de núcleo esteroide y flavonoide de los bulbos para la posterior identificación cualitativa de D. bulbifera cosechados en el piedemonte llanero de Colombia.

\section{Metodología}

\section{A. Características Generales}

El estudio se realizo en la zona rural de la ciudad de Villavicencio, Meta a una altitud entre los 410 y $450 \mathrm{msnm}$ en los predios de la Universidad de los Llanos. Se recolectaron muestras de bulbos de $D$. bulbifera entre los 22 a 25 días después de finalizada la inflorescencia en cuatro puntos geograficos con crecimiento silvestre de acuerdo con la Fig. 1. Para la recoleccion de bulbos se tuvieron en cuenta aspectos como: las condiciones ambientales, el tamaño, la formación y color de bulbos.

\section{B. Tratamiento Previo}

Los bulbos recolectados, fueron sometidos a un proceso de limpieza y desinfección por inmersión en agua y aspersión con etanol $70 \%$. Posteriormente se dejaron en exposición al sol por alrededor de 2 horas (solarización). Finalmente se hizo una reducción de tamaño para lograr trozos de $5 \mathrm{~mm}$ de lado tanto para la pulpa como para la cáscara de los bulbos. Lo anterior permitió realizar la maceración de los bulbos empleando tres solventes: agua, aceite mineral y etanol $40 \%$. 


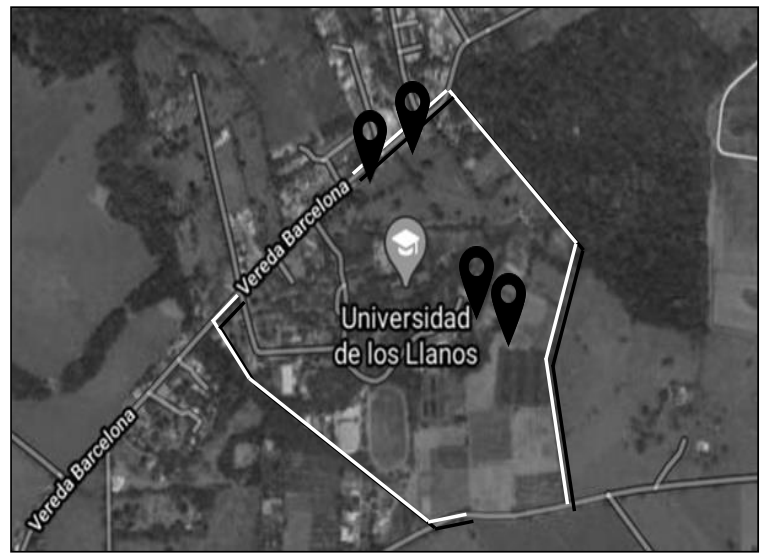

Fig. 1. Imagen satelital del área perteneciente a los predios de la Universidad de los Llanos, con marcadores de los puntos de cosecha de las muestras de $D$. bulbifera.

\section{Materiales y Métodos}

1) Maceración

La TABLA I muestra los reactivos y temperaturas empleados para la maceración de las muestras cortadas de $D$. bulbifera:

TABLA I

MACERADOS REALIZADOS

\begin{tabular}{|c|c|}
\hline \multicolumn{2}{|c|}{ Alcoholato } \\
\hline $1 \mathrm{Kg}$ de bulbos de D. bulbifera & 1 Litro solución de etanol al $40 \%$. \\
\hline \multicolumn{2}{|c|}{ Hidrolato } \\
\hline $1 \mathrm{Kg}$ de bulbos de $D$. bulbifera & 1 Litro de agua destilada a $50^{\circ} \mathrm{C}$ \\
\hline & Oleato \\
\hline $1 \mathrm{Kg}$ de bulbos de $D$. bulbifera & 1 Litro de aceite mineral a $51^{\circ} \mathrm{C}$. \\
\hline
\end{tabular}

Cada uno de los macerados se agitaron mecánicamente por 6 horas, posterior a ello, se trasvasaron a botellas de vidrio de color ámbar aforando a $750 \mathrm{ml}$ de extracto por cada solvente. Luego, bajo penumbra se dejaron almacenadas por un tiempo de 25 días [15]. Las condiciones de trabajo fueron constantes a $25^{\circ} \mathrm{C}$ y humedad relativa del $78 \%$.

\section{2) Detección de núcleos químicos}

El extracto obtenido de cada macerado se filtró y se sometió a pruebas de detección visual señaladas en la TABLA II, que identificaron el núcleo esteroidal o flavonoide mediante viraje de color.

Según lo expuesto en la práctica de glicósidos, la metodología utilizada de pruebas cualitativas (RxSh y RxL-B) fue ajustada y adaptada del protocolo ([15], [16]) para cada extracto de $D$. bulbifera,. La solución patrón de Flavonoides para la RxSh fue una dilución al $60 \%$ de vino de uva $\mathrm{La}$ Bodeguita ${ }^{\circledR}$, vino tinto dulce. El patrón de alcaloides para la RxL-B fue solución de hidrocortisona $1 \%$ con agua destilada. Las muestras para cada prueba se realizaron diluyendo el extracto del macerado 1:1 con el solvente respectivo, ejemplo: $20 \mathrm{ml}$ del alcoholato obtenido con $20 \mathrm{ml}$ de alcohol 40\%. Del cual se obtuvieron 6 muestras para cada extracto. El blanco es el mismo solvente, en la proporción antes descrita. Adicionalmente se estableció el testigo para las reacciones de detección, empleando directamente trozos triturados de $D$. bulbifera (pulpa y cáscara).

TABLA II

MATRIZ DE VALIDACIÓN DE PRUEBAS CUALITATIVAS (ADAPTADA Y AJUSTADA DE P. VÉlEZ Y S.C GRANJA [16], [17])

\begin{tabular}{|c|c|c|}
\hline Prueba & Positividad & Descripción \\
\hline $\begin{array}{c}\text { RxSh } \\
\mathbf{Z n} / \mathbf{H C l} \mathbf{~ c c})\end{array}$ & $\begin{array}{c}\text { Produce una } \\
\text { coloración rojiza } \\
\text { tenue hasta } \\
\text { escarlata }\end{array}$ & $\begin{array}{c}\text { El zinc en polvo reacciona con } \\
\text { HCl cc. El hidrogeno generado } \\
\text { produce por reducción el ion } \\
\text { flavilo de color rojo escarlata. }\end{array}$ \\
\hline & $\begin{array}{c}\text { Color verde, } \\
\text { azul petróleo } \\
\text { positiva para } \\
\text { esteroides; } \\
\text { amarillo naranja; } \\
\text { marrón pardo es } \\
\text { positiva para } \\
\text { triterpenos }\end{array}$ & $\begin{array}{c}\text { El lavado con cloroformo } \\
\text { remueve la fracción apolar, } \\
\text { luego es alcalinizado con el } \\
\text { anhidrido acético, la digestión } \\
\text { es con el ácido sulfúrico donde } \\
\text { se solubilizan nuevamente } \\
\text { formando un complejo } \\
\text { coloreado al enfriarse } \\
\text { lentamente. }\end{array}$ \\
\hline
\end{tabular}

El diseño experimental factorial elegido para obtener los resultados de investigación fue, 2 factores y 3 niveles $(2 \times 3)$ con 6 repeticiones, con un total de 36 ensayos, para identificar la presencia de núcleos esteroidales y flavonoides en extractos macerados del bulbo de D. bulbifera.

\section{3) Evaluación de las reacciones de detección}

La interpretación de los resultados obtenidos en las detecciones cualitativas se realizó mediante la triangulación de las observaciones [17]. Se asignó una valoración numérica de 1 a 4 como lo propone la Tabla III, donde 1 es una reacción igual a la del blanco, y 4 es una reacción igual a la del patrón químico definido para cada metabolito de interés: esteroideshidrocortisona, flavonoides- vino tinto.

TABLA III

NORMA DE CALIFICACIÓN DE LA REACCIÓN PARA LA DETECCIÓN
\begin{tabular}{|c|c|}
\hline Puntaje & Norma \\
\hline $\mathbf{1}$ & Se parece la reacción al blanco \\
\hline $\mathbf{2}$ & Hay una reacción que no es descrita como la positiva \\
\hline $\mathbf{3}$ & Reacción parecida al patrón, pero con distorsión \\
\hline $\mathbf{4}$ & Reacción igual al patrón \\
\hline
\end{tabular}

Se estableció una matriz de relaciones por color (TABLA IV.) en donde la pareja ordenada se organizó dejando en el eje vertical los puntajes asignados para flavonoides y en el eje horizontal los puntajes asignados a esteroides.

TABLA IV

MATRIZ DE RELACIONES PARA LA EVALUACIÓN DE LOS EXTRACTOS.

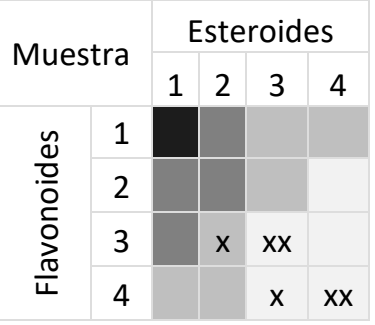


De esta manera el cruce de puntajes entre flavonoides y esteroides completó la matriz indicando la frecuencia acumulada de las 6 repeticiones en las detecciones realizadas. Esto permitió crear una escala de colores, cuyos criterios de valoración se relacionaron con la afinidad: solvente - núcleo, mostrada en la TABLA V.

TABLA V.

VALORACIÓN DE LA POLARIDAD DEL SOLVENTE SEGÚN EL GRUPO ASIGNADO POR EL COLOR DE LA MATRIZ DE RELACIONES.

\begin{tabular}{|c|c|}
\hline Cualificación & Descripción \\
\hline & $\begin{array}{c}\text { Polaridad de solvente muy afín al compuesto de interés } \\
\text { Polaridad semejante al compuesto de interés }\end{array}$ \\
\hline Poca afinidad del solvente con el compuesto de interés \\
\hline Compuesto de interés insoluble en el solvente \\
\hline
\end{tabular}

Bajo el mismo criterio de la matriz de relaciones se elaboró una matriz ponderada, con el fin de facilitar la identificación de la efectividad de la acción del solvente en la separación de los núcleos de interés.

\section{Resultados}

\section{1) Maceración}

Una extracción vegetal efectiva debe ser aquella en la que el solvente genera lisis de la pared celular, garantizando la conservación e integridad de los compuestos a extraer ([18] Pagina 12 - 17). De acuerdo con la información reportada en la TABLA VI. en la que se muestran las observaciones obtenidas para los procesos de separación de núcleos flavonoides y esteroides, luego de 25 días de maceración con tres solventes distintos, se evidenció que, para la maceración acuosa, la premisa anterior, no se llevó a cabo, en su lugar se generó fermentación de las muestras de $D$. bulbifera hacia el día cinco de iniciada la maceración.

La maceración oleaginosa tampoco presentó indicios de lisis celular por solvente. Por el contrario, la maceración alcohólica, indicó que la lisis fue efectiva, por lo que se obtuvieron resultados positivos en la identificación en los núcleos de interés.

TABLA VI.

\begin{tabular}{|c|c|c|c|c|c|}
\hline & Tipo & Color & Textura & $\begin{array}{c}\text { Aparienci } \\
\text { a }\end{array}$ & Aroma \\
\hline \multirow[b]{2}{*}{ Hidrolato } & Bulbo & Beige & $\begin{array}{l}\text { Blanda } \\
\text { cremosa }\end{array}$ & $\begin{array}{l}\text { Tersa, } \\
\text { suave }\end{array}$ & $\begin{array}{c}\text { Alcohólic } \\
\text { o }\end{array}$ \\
\hline & $\underline{\text { Solvente }}$ & Beige & $\begin{array}{c}\text { Aument } \\
\text { o } \\
\text { viscosid } \\
\text { ad }\end{array}$ & $\begin{array}{c}\text { Turbidez y } \\
\text { opalescenc } \\
\text { ia }\end{array}$ & $\begin{array}{c}\text { Frutos } \\
\text { secos y } \\
\text { pasa de } \\
\text { uva }\end{array}$ \\
\hline \multirow[b]{2}{*}{ Oleato } & Bulbo & Amarillo & $\begin{array}{l}\text { Firme, } \\
\text { intacto }\end{array}$ & Manchas & $\begin{array}{c}\text { No se } \\
\text { identifica }\end{array}$ \\
\hline & $\underline{\text { Solvente }}$ & Transparente. & $\begin{array}{l}\text { Fluido, } \\
\text { transluci } \\
\text { do }\end{array}$ & Terso & $\begin{array}{l}\text { No se } \\
\text { percibe }\end{array}$ \\
\hline \multirow[t]{2}{*}{ Alcoholato } & Bulbo & Café pardo & $\begin{array}{c}\text { Hinchad } \\
o, \\
\text { blando }\end{array}$ & $\begin{array}{l}\text { Firmeza } \\
\text { reducida }\end{array}$ & $\begin{array}{c}\text { Alcohólic } \\
\text { o }\end{array}$ \\
\hline & $\underline{\text { Solvente }}$ & Naranja oscuro & Turbio & $\begin{array}{l}\text { Tersa y } \\
\text { fluida }\end{array}$ & $\begin{array}{c}\text { Nueces y } \\
\text { tierra }\end{array}$ \\
\hline
\end{tabular}

La fermentación alcohólica generada en el macerado acuoso, sucedió a partir de las condiciones favorables de temperatura, humedad y concentracion de sustrato (almidón de la pulpa)[19] para microorganismos, que posiblemente se depositaron en el tiempo de solarización directa. El pardeamiento leve observado en los trozos de bulbo en el oleato, indicaron un efecto conservante aparente, resaltando el carácter hidrofóbico del solvente sobre los núcleos de interés.

\section{2) Detección de núcleos químicos}

En la Tabla VII., se aprecian los puntajes obtenidos de la triangulación de observación, para la detección de los núcleos flavonoides y esteroides en cada uno de los extractos producto de las maceraciones sobre los bulbos de D. bulbifera, siguiendo los criterios de la Tabla III.

TABLA VII.

PUNTAJES DE LA TRIANGULACIÓN DE OBSERVACIÓN PARA EXTRACTOS DE $D$. bulbifera.

\begin{tabular}{|c|c|c|c|c|}
\hline \multicolumn{5}{|c|}{ Glicoalcaloides } \\
\hline Repetición & Bulbo crudo & Hidrolato & Oleato & Alcoholatura \\
\hline $\mathbf{1}$ & 3 & 2 & 1 & 4 \\
\hline $\mathbf{2}$ & 3 & 2 & 1 & 3 \\
\hline $\mathbf{3}$ & 3 & 1 & 2 & 4 \\
\hline $\mathbf{4}$ & 2 & 2 & 1 & 4 \\
\hline $\mathbf{5}$ & 3 & 2 & 1 & 4 \\
\hline $\mathbf{6}$ & 3 & 1 & 1 & 4 \\
\hline Resultado & $\mathbf{3} \pm \mathbf{0 , 4 1}$ & $\mathbf{2} \pm \mathbf{0 , 5 2}$ & $\mathbf{2} \pm \mathbf{0 , 4 1}$ & $\mathbf{4} \pm \mathbf{0 , 4 1}$ \\
\hline
\end{tabular}

\begin{tabular}{|c|c|c|c|c|}
\multicolumn{7}{|c|}{ TABLA VII (CONTINUACIÓN) } \\
\hline $\mathbf{1}$ & 3 & 1 & 1 & 3 \\
\hline $\mathbf{2}$ & 3 & 2 & 2 & 4 \\
\hline $\mathbf{3}$ & 2 & 1 & 1 & 3 \\
\hline $\mathbf{4}$ & 3 & 2 & 1 & 4 \\
\hline $\mathbf{5}$ & 2 & 1 & 1 & 4 \\
\hline $\mathbf{6}$ & 2 & 1 & 2 & 4 \\
\hline Resultado & $\mathbf{3} \pm \mathbf{0 , 5 5}$ & $\mathbf{2} \pm \mathbf{0 , 5 2}$ & $\mathbf{2} \pm \mathbf{0 , 5 2}$ & $\mathbf{4} \pm \mathbf{0 , 5 2}$ \\
\hline
\end{tabular}

Con un promedio superior a 3 puntos en las pruebas realizadas en el bulbo crudo, se pudo inferir que los compuestos de interés se encuentran disponibles en la cáscara y en la pulpa de $D$. bulbifera, de manera que las detecciones realizadas a los diferentes extractos sean parecidos en un $83 \%$ al patrón de esteroides y $50 \%$ al patrón de flavonoides (Fig. 2). Esto evidenciaría por un lado la acción de lisis de la pared celular, realizada por los solventes y por otro justifica la distorsión de los resultados en las pruebas de detección realizadas, ya que la extracción con un ácido fuerte como el ácido sulfúrico $3,5 \mathrm{M}$ en la prueba RxL-B favorece tanto la extracción como la hidrólisis simultáneas[20], manifestándose mediante la aparición de coloraciones de oscuras en la superficie marcada al parecer por el contenido de almidón de la pulpa que contiene gomas y almidones, que al combinarse con anhidridos y luego bajar su $\mathrm{pH}$ rápidamente tienden a generar coloraciones ocres y oscuras [21].

\section{3) Evaluación de las reacciones de detección}

El promedio de 4 puntos obtenido en ambas pruebas para el alcoholato demostró que la polaridad del etanol al $40 \%$ es el solvente de mayor afinidad a los compuestos flavonoides y glicoalcaloides de D. bulbifera esto corresponde a $67 \%$ y $83 \%$ respectivamente de acuerdo con lo expuesto en la Fig 2. 


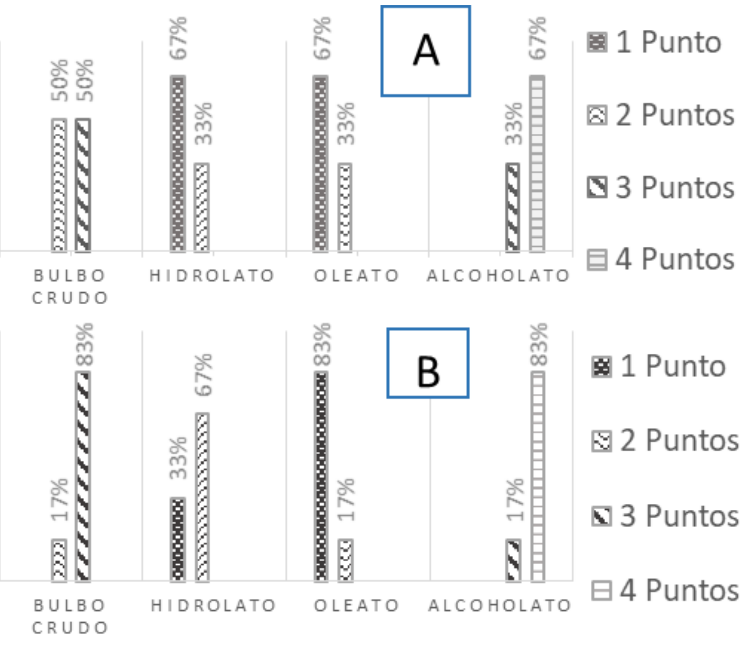

Fig. 2. Porcentajes de detección para cada núcleo en cada uno de los extractos, estos denotan el comportamiento de los solventes para la RxSh y RxL-B. Punto 1, 2, 3, 4 corresponden a la norma de calificación de la Tabla III.

A) Porcentaje de detección de flavonoides por el método de viraje de color de la RxSh.

B) Porcentaje de detección de esteroides por el método de viraje de color de la RxL-B.

TABLA VIII

MATRIZ DE RELACIONES DE LA AFINIDAD DE LOS EXTRACTOS FRENTE A PRUEBAS DE DETECCIÓN.

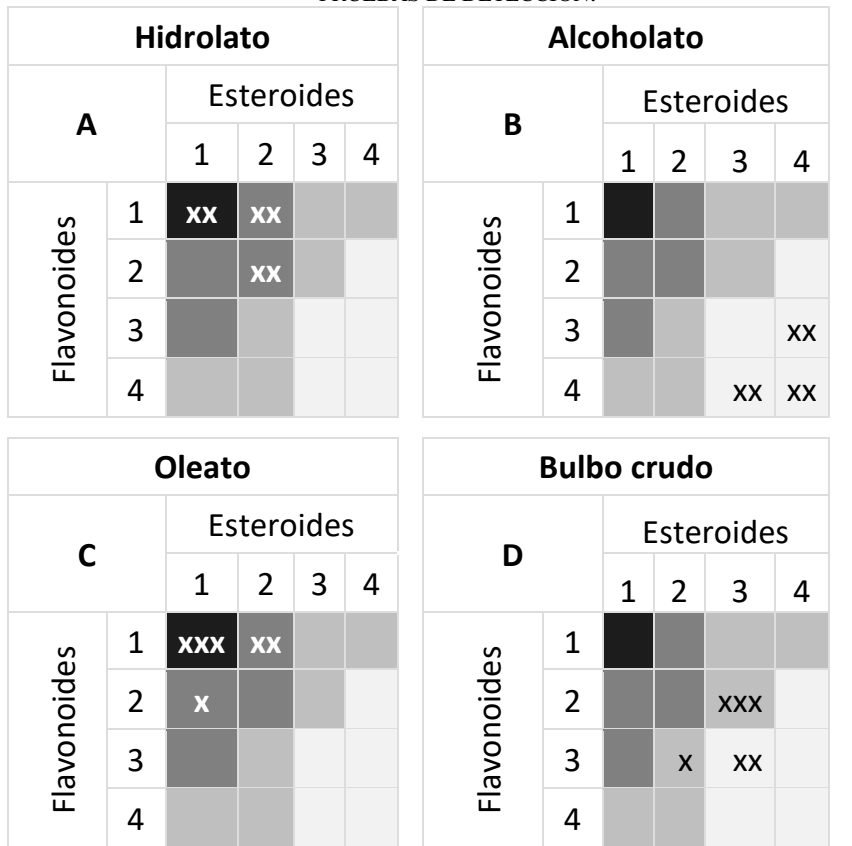

Los puntajes consolidados para cada uno de los extractos se distribuyen así: A) Hidrolato, B) Alcoholato, C) Oleato, D) Testigo: Bulbo crudo. La "X" representa el resultado de un cruce de puntajes para cada repetición evaluada. Ejemplo: en la tabla $\mathrm{C}$ una repetición obtuvo un puntaje de 1 para esteroides y 2 para flavonoides

La detección de los metabolitos alcaloides y flavonoides en el oleato presentó una reacción similar al blanco de hidrocortisona $1 \%$ y solución de vino tinto en todas las repeticiones cuyo puntaje de norma es 1 (Tabla VII), indicando reacciones negativas de detección en el $83 \%$ para alcaloides y $67 \%$ para flavonoides (Fig. 2).
En la tabla VIII, se observan las matrices que consolidan la combinación de los resultados de las pruebas cualitativas de detección, donde según la afinidad del solvente con el compuesto químico de interés son clasificados por colores.

La matriz A de la tabla VIII, arrojó que el agua empleada en la separación, es insoluble y poco afín con los metabolitos de interés, lo que corresponde al $67 \%$ según lo expuesto en la matriz ponderada (Tabla IXTABLA IX), puesto que la fermentación ocasionada por la dispersión del almidón en agua, enmascaró la posible concentración de los compuestos con núcleos de interés liberados por la lisis que este solvente causó en la pared celular de los trozos de D. bulbifera. Esto abrió la posibilidad, para considerar que se llevó a cabo una competencia por el agua libre entre los microorganismos contaminantes en las muestras y la solubilidad de los compuestos, contrario al efecto biocida atribuido a los glicoalcaloides propios de la familia Dioscórea [22].

A partir de los resultados de la matriz C de la tabla VIII, se pudo afirmar que el aceite mineral tuvo una valoración similar al solvente anteriormente descrito, pero representando el 50\% de insolubilidad del solvente (Tabla IX). Una vez finalizado el tiempo de maceración, los trozos de la muestra presentaron los atributos sensoriales mostradas en la tabla VITABLA VI, en los que se demuestra la integridad de los mismos, confiriéndole al aceite mineral propiedades conservantes.

Por lo observado en la matriz B de la tabla VIII, se pudo deducir que la polaridad del alcohol al $40 \%$ es afín a los compuestos esteroides y flavonoides presentes en trozos de bulbo en el $100 \%$ de los casos (Tabla IX), siendo confirmado por otros estudios realizados con extractos de D. bulbifera con solventes como el metanol y cloroformo [11]. Sin embargo la precisión en la confirmación de la RxSh para flavonoides de D. bulbifera, presentó interferentes relacionados con la aparición de compuestos lechosos o blancuzcos que se asocian con la presencia de flavonoides como chalconas, auronas o isoflavonas [23], [15].

TABLA IX.

MATRIZ PONDERADA DE LA EVALUACIÓN DE LOS SOLVENTES PARA LA EXTRACCIÓN DE NÚCLEOS ESTEROIDES Y FLAVONOIDES.

\begin{tabular}{|c|c|c|c|c|}
\hline \multicolumn{4}{|c|}{ Solvente } & \multirow{3}{*}{ Determinación } \\
\hline $\begin{array}{l}\text { Agua } \\
\text { Destilada }\end{array}$ & $\begin{array}{l}\text { Aceite } \\
\text { mineral }\end{array}$ & $\begin{array}{l}\text { Alcohol } \\
\text { etílico } 40 \%\end{array}$ & \multirow{2}{*}{$\begin{array}{l}\text { Bulbo } \\
\text { Crudo }\end{array}$} & \\
\hline \multirow[t]{3}{*}{ Hidrolato } & Oleato & Alcoholato & & \\
\hline & & $100 \%$ & $33 \%$ & $\begin{array}{c}\text { Polaridad de solvente muy afín al } \\
\text { compuesto de interés }\end{array}$ \\
\hline & & & $67 \%$ & $\begin{array}{c}\text { Polaridad semejante al compuesto } \\
\text { de interés }\end{array}$ \\
\hline $67 \%$ & $50 \%$ & & & $\begin{array}{l}\text { Poca afinidad del solvente con el } \\
\text { compuesto de interés }\end{array}$ \\
\hline $33 \%$ & $50 \%$ & & & $\begin{array}{l}\text { Compuesto de interés insoluble } \\
\text { en el solvente }\end{array}$ \\
\hline
\end{tabular}

\section{CONCLUSIONES}

El alcohol es el solvente que extrae de forma efectiva metabolitos secundarios de núcleos esteroides y flavonoides durante la maceración de bulbos de D. bulbifera, ya que en las pruebas de detección (RxL-B y RxSh) para estos núcleos presentó un $100 \%$ de afinidad, dado su carácter hidrofílico y comparando el contenido de su extracto con lo hallado en el bulbo crudo. 
El efecto biocida de los compuestos esteroidales como los glicoalcaloides de $D$. bulbifera impiden obtener un macerado acuoso con agua destilada por 25 días, debido a que se genera fermentación después del quinto día por microorganismos, principal interferente para no detectar flavonoides en este extracto.

El aceite mineral se comportó como un agente conservante debido a su carácter hidrofóbico opuesto a la naturaleza de los núcleos de interés en los trozos de $D$. bulbifera, por lo tanto, la cantidad de compuestos con núcleo esteroidal o flavonoide no pudo ser detectada por las pruebas aplicadas al extracto.

La capacidad antioxidante de los flavonoides y la posibilidad de extraer esteroides de la pulpa y la cáscara detectados en $D$. bulbifera, permite considerar la idea de usar estos bulbos como una nueva materia prima en el sector agroindustrial.

\section{RECOMENDACIONES}

Se propone plantear el aislamiento de compuestos con núcleos esteroides, empleando algunas metodologías propuestas en la referencia [24], con el fin de cuantificar el contenido en bulbos de $D$. bulbifera extraído en solventes alcohólicos.

Teniendo presente que los núcleos de interés son de carácter hidrofílico, se sugiere realizar extracciones con otros solventes de esta naturaleza como el ácido acético al $5 \% \mathrm{v} / \mathrm{v}$, mezclas de este con etanol y cloroformo. [25]

Se podría establecer en próximas investigaciones, un objetivo que permita identificar la fracción de bulbo donde se acumulan los grupos esteroide y flavonoide [26], para evidenciar en qué segmento transversal del bulbo existe más o menos concentración, proponiendo la creación de un protocolo de cuantificación adaptado a D. bulbifera, llevado a cabo mediante HPLC [27].

\section{AGRADECIMIENTOS}

Para la ejecución de este proyecto y en especial la producción de este articulo agradecemos a los docentes: MsC María Cristina Ospina, MsC. Luis Gilberto López Muñoz e Ingeniera María Alejandra Cruz Domínguez de la Universidad de los Llanos por la gestión realizada para el grupo investigador; a la profesora Ingeniera Ph.D. María Patricia Rodríguez de la Universidad de los Llanos por su dedicación, a la Licenciada Ph.D. Giovanna del Pilar Fuentes Medina de la Universidad Nacional, sede Bogotá D.C. por su disponibilidad, constancia y esfuerzo en cada etapa de este proceso; y a la Universidad de los Llanos por brindarnos la oportunidad y el espacio para adelantar este proyecto de investigación.

\section{REFERENCIAS}

[1] G. Gakpetor, "The Cost Effectiveness of Biological Control Of Air Potato Vine Using Air Potato Beetle; A Biocontrol Agent In Florida", A thesis Submitt. Partial fulfillment Requir. degree master's Sci. Agric. Sci. Coll. Agric. Food Sci. Florida Agric. Mech. Univ., vol. 8, núm. 5, p. 55,2019 .

[2] J. Ikwebe, M. H. Mayel, E. Mamman, S. V Tatah, y S. S. Adebayo, "Impact Of Processing Methods On The Levels Of Iron, Zinc And
Magnesium In Yam (Dioscorea Species)", FUW Trends Sci. Technol. J., vol. 5, núm. 2, pp. 538-541, 2020.

[3] E. J. Price, P. Wilkin, V. Sarasan, y P. D. Fraser, "Metabolite profiling of Dioscorea (yam) species reveals underutilised biodiversity and renewable sources for high-value compounds", Sci. Rep., vol. 6, núm. July, pp. 1-10, 2016. DOI: 10.1038/srep29136

[4] D. G. Omayio, G. O. Abong, y M. W. Okoth, "A Review of Occurrence of Glycoalkaloids in Potato and Potato Products A Review of Occurrence of Glycoalkaloids in Potato and Potato Products", núm. December 2016. DOI: http://dx.doi.org/10.12944/CRNFSJ.4.3.05

[5] M. Khalid, Saeed-ur-Rahman, M. Bilal, y D. feng HUANG, "Role of flavonoids in plant interactions with the environment and against human pathogens - A review", J. Integr. Agric., vol. 18, núm. 1, pp. 211-230, 2019. DOI: 10.1016/S2095-3119(19)62555-4

[6] M. L. McKoy, K. Grant, H. Asemota, O. Simon, y F. Omoruyi, "Renal and hepatic function in hypercholesterolemic rats fed Jamaican Bitter Yam (Dioscorea polygonoides)", J. Diet. Suppl., vol. 12, núm. 2, pp. 173-183, 2015. DOI: 10.3109/19390211.2014.952860

[7] S. J. Maleki, J. F. Crespo, y B. Cabanillas, "Anti-inflammatory effects of flavonoids", Food Chem., vol. 299, núm. March 2019. DOI: 10.1016/j.foodchem.2019.125124

[8] F. Rena, K. Reillya, M. Gaffneya, J. P. Kerry, M. Hossaina, y D. K. Raia, "Evaluation of polyphenolic content and antioxidant activity in two onion varieties grown under organic and conventional production systems", Org. Conv. Onion AUTHORS, vol. 28, núm. 3, pp. 303-325, 2017. DOI: $10.1002 /$ jsfa. 8138

[9] J. A. Forero, L. I. Sotelo Díaz, y L. E. Díaz, "Estudio Del Ruto Del Borojo Para La Obtención De Compuestos De Interes En La Industria Cosmetologica Alimenticia: "Efecto de extractos fenólicos de Borojó (Borojoa patinoi cuatrec) en dos estados de maduración sobre la actividad in vitro de la enzima Hialuronidasa".,, Biblioteca Octavio Arizmendi Posada Universidad De La Sabana Chía - Cundinamarca", Universidad de la Sabana, 2013.

[10] X. R. Guan, L. Zhu, Z. G. Xiao, Y. L. Zhang, H. B. Chen, y T. Yi, "Bioactivity, toxicity and detoxification assessment of Dioscorea bulbifera L.: a comprehensive review", Phytochem. Rev., vol. 16, núm. 3, pp. 573-601, 2017. DOI: 10.1007/s11101-017-9505-5

[11] R. M. Nur, L. H. Nugroho, R. M. Nur, y L. H. Nu-, "Cytotoxic Activities of Fractions from Dioscorea bulbifera L. Chloroform and Methanol Extracts on T47D Breast Cancer Cells", vol. 10, núm. 1, pp. 33-38, 2018. DOI: 10.5530/pj.2018.1.7

[12] T. Cañon y M. Menco, "Estudio Fitoquímico De La Especie Vegetal Solanum Crinitipes Dunal (Solanaceae) Y Evaluación De Uso Como Agente Antimicrobiano", J. Chem. Inf. Model., vol. 53, núm. 9, pp. 1689-1699, 2018.

[13] M. Jiménez-Montero y A. A. Martínez, "Estudio Etnobotánico De La Papa De Aire (Dioscorea Bulbifera L.) En Donoso (Colón, República De Panamá)", Luna Azul ISSN 1909-2474, núm. 42, pp. 54-67, 2016. DOI: 10.17151/luaz.2016.42.6

[14] F. M. Niyas, "Medicinal uses of Dioscorea bulbifera- A Review", Res. J. Pharm. Technol., vol. 8, núm. 8, pp. 1059-1062, 2015. DOI: 10.5958/0974-360X.2015.00182.1

[15] P. Vélez y M. F. Pilaquinga, "Extracción e identificación de solanina obtenida de berenjena". quito, Ecuador, pp. 1-12, 2016.

[16] S. C. Granja, "Síntesis de nanopartículas de plata utilizando como agente reductor los flavonoides, polifenoles y azúcares reductores presentes en el extracto acuoso de las hojas de Baccharis latifolia (Chilca)", Pontificia Universidad Católica Del Ecuador, 2019.

[17] M. A. Aya, K. N. Benjumea, M. I. Gómez, L. J. Laguna, W. D. Medina, y K. T. Silva, "Métodos Cualitativos- al alcance de tu mano, teoría, fundamentos y estudio de casos", Universidad Santo Tomas, Sede Villavicencio, 2020.

[18] I. J. Torres Romero, "Análisis metodológico para la extracción de ADN metagenómico en muestras coprológicas humanas", 2018.

[19] K. Shirai y F. Malpica, "Tecnología de Fermentaciones Alimentarias", Univ. Autónoma Metrop. Unidad Iztapalapa, vol. 13, núm. 186, p. 115, 2013.

[20] M. Jiménez-montero, S. Sánchez, y F. Toabré, "Evaluación nutricional de la papa de aire (Dioscorea bulbifera L.) cultivada en Panamá", vol. 67, 2017.

[21] L. Giannuzzi, “Toxicología general y aplicada”, Toxicol. Gen. y Apl., 2020.

[22] D. R. Gordon, "Effects of Invasive, Non-Indigenous Plant Species On Ecosystem Processes: Lessons From Florida", Inorg. Chem., vol. 44, 
núm. $\quad 7, \quad$ pp. 2459-2464, 2005. DOI: 10.1890/10510761(1998)008[0975:EOINIP]2.0.CO;2

[23] S. S. Li, I. A. Iliya, J. Z. Deng, y S. X. Zhao, "[Flavonoids and anthraquinone from Dioscorea bulbifera L.]", Zhongguo Zhong Yao Za Zhi, vol. 25, núm. 3, p. 159-160, 2000.

[24] L. D. López Quimbayo, "Extracción a partir de hojas y semillas de Pentacalia nítida y evaluación de la actividad antimicrobiana del extracto proteico acuoso", pp. 5, 9-11, 2012.

[25] M. S. Mokbel y F. Hashinaga, "Antibacterial and Antioxidant Activities of Banana (Musa, AAA cv. Cavendish) Fruits Peel", Am. J. Biochem. Biotechnol., vol. 1, núm. 3, pp. 125-131, 2005. DOI: 10.3844/ajbbsp.2006.125.131

[26] W. Peña, "Evaluación del contenido de glicoalcaloides en el pelado, cocción y fritura de variedades de papa nativa", Escuela Politécnica Nacional, 2011.

[27] M. Friedman, J. N. Roitman, y N. Kozukue, "Glycoalkaloid and calystegine contents of eight potato cultivars", J. Agric. Food Chem., vol. 51, núm. 10, pp. 2964-2973, 2003. DOI: 10.1021/jf021146f.

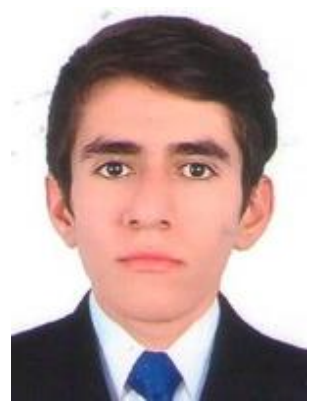

Diego Alejandro Bejarano Navas. Nació en Villavicencio-Meta, Colombia el 17 de febrero de 1997. Se graduó en el colegio Instituto Técnico Industrial, culminó sus estudios en Ingeniería Agroindustrial en la Universidad de los Llanos. Ejerce como joven investigador desde el 2018 en el Grupo de Investigación en Ciencia, Tecnología e Innovación Agroindustrial CITIA de la Universidad de los Llanos. Entre sus campos de interés están la fito- química y microbiología.

Participó en el V Encuentro de Agroindustria e Innovación en la Universidad del Atlántico, recibió títulos en curso técnico intensivo sobre el "Manejo Integrado del Cultivo del Cacao" por parte de FEDECACAO; "Multiplicación y Propagación de Material Vegetal", "Aplicación de Conceptos básicos de la Gestión de Inocuidad HACCP con Enfasis en los Procesos de Validación y Verificación", "Aplicación de Buenas Prácticas de Manufactura en la Industria de Alimentos y Bebidas", e "Higiene y Manipulación de Alimentos" otorgados por el Servicio Nacional de Aprendizaje (SENA). En 2020 obtuvo grado meritorio por su participación en el desarrollo y consolidación en el trabajo de investigación "Evaluación del contenido de glicoalcaloides y flavonoides totales con diferentes tratamientos de "papa de aire" (Dioscórea bulbifera Salv.) cosechados en los llanos orientales de Colombia". En la actualidad lidera y gestiona procesos asociados al diseño e implementación de estrategias que fomenten el emprendimiento regional.

ORCID: https://orcid.org/0000-0003-4521-315X.

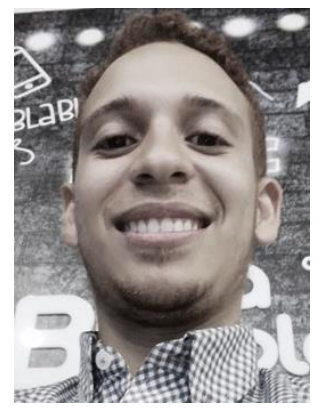

Jhon Esteban Armenta Roncancio. Nació en Bogotá D.C., Colombia el 30 de agosto 1992, finalizo sus estudios en la ciudad de Villavicencio en la Institución Educativa John F. Kennedy, estudió cocina profesional en el Servicio Nacional de Aprendizaje SENA de Colombia y estudió en la Universidad de los Llanos.

Dentro del campo de la ingeniería se destaca como profesional de alto valor en el área de investigación y desarrollo agroindustrial, realizando procesos de generación de conocimiento en Medellín, San Vicente de Chucuri y en el Instituto de Acuicultura de los Llanos IALL, además pertenece desde el año 2018 al Grupo de Investigación en Ciencia Tecnología E Innovación Agroindustrial. CITIA. En 2020 obtuvo grado meritorio por su participación en el desarrollo y consolidación de trabajo de investigación "Evaluación del contenido de glicoalcaloides y flavonoides totales con diferentes tratamientos de "papa de aire" (Dioscórea bulbifera Salv.) cosechados en los llanos orientales de Colombia". actualmente se desempeña como líder de innovación en biotecnología de primera generación.

ORCID: https://orcid.org/0000-0002-8421-2590.

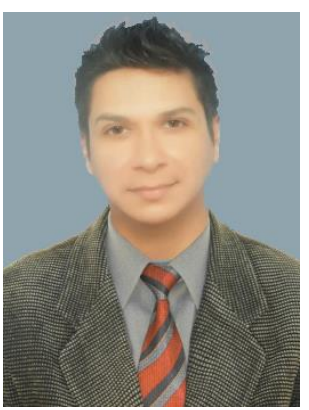

Luis Gilberto López Muñoz. Nació en Fusagasugá- Cundinamarca el 22 noviembre de 1979, graduado como bachiller académico del colegio Diocesano Ricaurte la Salle en el año de 1994 en Fusagasugá, posteriormente se radica en la ciudad de Villavicencio donde estudia en la Universidad del Meta, en el programa de Ingeniería Agroindustrial, título que obtiene en el año 2002 y en el año 2019 obtiene el título en la maestría en estudios en desarrollo local otorgado por la Universidad de los llanos.

Ejerció profesionalmente en diferentes empresas como Fanagra, Colciencias, Alcaldía de Villavicencio, Gobernación del Meta, Servicio Nacional de Aprendizaje sede Hachón y Universidad de los Llanos. Apasionado por la investigación especialmente en la transformación de plantas medicinales y condimentarías, en la actualidad, se dedica a la docencia en la Universidad de los llanos en la ciudad de Villavicencio como docente tiempo completo, dictando las materias de Metodología de la Investigación, Profundización y Procesos No Alimentarios.

ORCID: https://orcid.org/0000-0003-3186-8577.

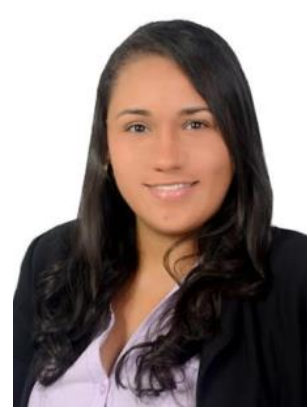

María Alejandra Cruz Domínguez. Nació en Villavicencio, Colombia, el 16 de junio de 1993. Se graduó del Colegio Nacionalizado Femenino de Villavicencio, estudió en la Universidad de los Llanos y obtuvo el título de Ingeniera Agroindustrial. Realizó su práctica profesional en el laboratorio de Plantas Térmicas y Energías Renovables en la Universidad Nacional de Colombia Sede Bogotá, se ha desempeñado como docente catedrática en la Universidad de los Llanos y como profesional de apoyo en la elaboración del PERS Orinoquía con el Observatorio del Territorio de la Universidad de los Llanos. En el año 2016 la Ingeniera Cruz fue exaltada en la noche de la excelencia de la Universidad de los Llanos por la publicación del artículo "Efecto de la temperatura en el potencial de aprovechamiento energético de los productos del pirólisis del cuesco de palma", en 2015, el cuál fue publicado en revista indexada en febrero de 2016 y mejor promedio de la carrera del programa de Ingeniería Agroindustrial. ORCID: https://orcid.org/0000-0002-3122-132X. 